\title{
RELATIONSHIPS WITH THE MEDICAL STAFF AND ASPECTS OF SATISFACTION WITH CARE EXPRESSED BY PARENTS OF CHILDREN WITH CANCER
}

\author{
Oscar A. Barbarin \\ Mark A. Chesler
}

\begin{abstract}
Seventy-four parents of children with cancer were asked to characterize the behavior of medical staff members with whom they interacted. Seven empirically distinct dimensions of staff behavior relevant to their relationships with parents were identified, including information transmission, clarity and honesty of communication, acceptance of parental efficacy, resolution of conflicts, personal contact with parents, empathy with the child, and staff competence. With respect to their experiences with the treatment of their child, parents also were asked to indicate their satisfaction with the medical staff in terms of changes in their respect and/or anger for the medical staff, changes in feelings about doctors, support received from doctors and nurses, and stress resulting from tense relations with the staff. The seven dimensions of parent-staff relationships were used as predictors in a series of multiple regressions employing these satisfaction measures as criteria. The overall quality of the parent-staff relationship was best predicted by positive personal contact. The strongest predictor of whether or not parents felt increased anger was staff empathy with child. Increased respect for the medical staff was predicted by a combination of information transmission and perception of staff competence. Experience of support by parents was best predicted by information transmission and staff acceptance of parental efficacy in treatment and decision making. This complex pattern supports the usefulness of disaggregating measures of staff behavior and parent satisfaction when examining the relations between medical consumers and service providers.
\end{abstract}

Relationships between the medical care staff and their patients are thought to influence, at least indirectly, the quality of health care. In addition, the quality of these relationships appears to determine how patients and their families evaluate their experiences in medical care organizations. Although medical professionals are uniformly committed to high-quality medical practice, they may be less certain about the relevance of good in-

Address request for reprints to Oscar Barbarin, Family Development Project Lab, Department of Psychology, University of Michigan, 580 Union Drive, Ann Arbor, Michigan 48109.

The data were gathered with the participation and support of a major university medical center and children's hospital, and a parent self-help organization, SHARE: Families of Children with Cancer. This report, and the larger study, is part of an action-research effort conducted jointly by SHARE, the hospital staff, and the investigators. 
terpersonal relations to this concern. Moreover, they may be less prepared and able to create high-quality relationships.

In the midst of this uncertainty, attention to the quality of staff-patient relationships often is justified on the grounds that patient satisfaction mediates compliance with the medical regimen. A doctor's concern with patient feelings thus may be justified by the need to understand how to manage patients so that they follow through on therapeutic instructions and procedures, and so enhance their physical well-being. Becker and Maiman ${ }^{1}$ exhaustively review research that provides the empirical grounds for the link between staff-patient relationships and patient compliance. Several studies they cite show that when staff-patient relationships are good and communication is clear, patients are more likely to be active in clarifying instructions prerequisite to carrying out the treatment plan. In addition, good relationships are associated with high levels of trust and confidence between patient and staff, which are likely to be translated into faithful adherence to treatment procedures. Even when disagreements arise, a foundation of mutual respect and support may make it easier to resolve conflicts in a harmonious and equitable manner.

Recently research on medical care practice also has begun to value staff-patient interaction independent of its link to patient compliance. This body of work has tended to view patient satisfaction as important in its own right. In part, the value attached to patient satisfaction reflects an increasing endorsement by medical professionals of the link between emotional wellbeing and physical well-being. ${ }^{2}$ Therefore attention to staff-patient and stafffamily relations becomes an important facet of good medical practice. Consistent with this trend, DiMatteo and colleagues ${ }^{3,4}$ have conducted a series of studies that examine the relationship between patient-staff relationships and satisfaction with medical care.

Much of the existing research has used the concept of patient satisfaction to indicate favorableness of evaluation. For example, DiMatteo and Hays $^{3}$ assess satisfaction with physicians in terms of patients' liking of the physician and their willingness to return to the same physician or to recommend the physician to a friend. However, satisfaction is a complex phenomenon, and there is a myriad of component or related reactions that contribute to global evaluations. Exclusive use of global measures of satisfaction may mask interesting and important differences. For example, respect, gratitude, awkwardness, and anger may coexist within the same relationship. Some patients may experience anger or tension in response to medical staff members who are aloof and autocratic, and at the same time be deferent and have respect for their technical skills and social status. In such cases individuals may rate the medical staff favorably along a technical dimension but consider them inadequate with respect to an affective or interpersonal dimension. If global measures of satisfaction alone are used, important information 
about patients' reactions to medical care is lost. When undifferentiated, these potentially divergent inputs will have an unmeasured effect on global reports and may obscure the link between specific aspects of staff behavior and specific aspects of patient satisfaction. If we can distinguish among these diverse outcomes, we may be able to trace more definitively the pathways that connect specific staff behaviors to specific patient reactions.

The complexity of patient satisfaction thus requires use of a broader array of reactions than normally included in prior measurements. The array of reactions selected for this study includes "liking," "anger," "respect," "tenseness," and "support" because they tap interpersonal and technical arenas most central to patients' perceptions and evaluations of medical care.

Like satisfaction, relationships with the medical staff are complex and cannot be understood adequately in global terms alone. Several studies have begun to specify dimensions of relationships between patients and medical staff which may influence satisfaction. For example, Kulka, Cupper, and Cassell ${ }^{5}$ and Davis ${ }^{6}$ show that physicians' ability to communicate information and engage in meaningful interpersonal relationships with patients contribute significantly to patients' reports of satisfaction with health care services. DiMatteo and Hayes ${ }^{3}$ find significant relationships between patients' satisfaction and their assessment of physicians' effectivenss at communication and the quality of their interpersonal affectivity. Moreover, acceptance or active promotion of patient efficacy appears in several reports to affect the doctorpatient relationship and perhaps indirectly to improve the quality of health care. For example, Schulman ${ }^{7}$ reports that hypertensive patients who were treated as active participants, monitoring their physical condition and involved in decision making regarding treatment, were more effective in controlling their blood pressure than patients treated as passive recipients of care.

In the special circumstance when children are patients, Becker and Maiman $^{1}$ cite research supporting the importance of the relationship between physicians and parents in mediating compliance with medical treatment. Francis, Korsch, and Morris ${ }^{8}$ also report that mothers are more likely to comply with a course of treatment for their child when the physician is friendly, understanding, and warm; when the physician attends to specific parental concerns about the child; and when the staff takes the time to explain in some detail the meaning of diagnosis. Similar behaviors on the part of nurses were identified in Rath's study of mothers of chronically ill children. ${ }^{9}$ On the basis of these and other studies, Becker and Maiman ${ }^{1}$ conclude that poor staffpatient-family relationships, characterized by impersonal brief encounters, formal rejecting attitudes of physician towards the patient, failure to provide feedback after extensive interviews, and interpersonal conflicts, have a deleterious effect on patient compliance.

Chesler and Barbarin ${ }^{10,11}$ have specified these behaviors further in their analyses of detailed reports by parents of children regarding their relationships with medical staff members. On the basis of these reports, they identify 
several key dimensions that seem to capture the most salient ways parents characterized their relationships with the staff. These dimensions include (a) information transmission by staff, (b) honesty and clarity of staff communication, (c) staff acceptance of parental efficacy, (d) resolution of conflict, (e) staff empathy with the child, (f) quality of the staff's interpersonal contact with parents, and $(\mathrm{g})$ staff competence.

Although staff-patient and staff-parent relationships may influence the experiences and reactions of medical care consumers, the nature of this interaction or influence pattern is not well understood. For instance, we do not know which aspects of staff-patient-parent relationships produce which positive or negative perceptions or evalutions of care. This study assesses the influences of the seven dimensions of parent-staff relations identified by Chesler and Barbarin ${ }^{10}$ on several distinct aspects of parents' reactions to the medical care of their children with cancer. Our goal is to identify potential links between specific dimensions of staff behavior and important indicators of parental satisfaction: we expect that the specific dimensions will differ in the extent to which they relate to the diverse outcome measures. If global discussions of "good staff behaviors" and "parental satisfaction" can be made more specific, all parties may be better able to understand and influence their relationships in more positive directions.

\section{METHOD}

\section{Participants}

Informants for the study were drawn on a representative basis from a pool of 200 families of children with cancer treated over the past 5 years at a major university hospital center. Over 75 percent of the families contacted for an interview agreed to participate in the study. The data reported here are taken from interviews and questionnaires with 74 parents of surviving children with cancer. Of this group, approximately 20 percent were parents of survivng children under six; 40 percent were parents of surviving children between six and eleven; and 40 percent were parents of surviving children between eleven and twenty-one. With respect to diagnosis, children in participating families were broadly representative of the pediatric oncology population treated at the university center. Of those interviewed, 32 percent were parents of children with leukemia; 16 percent were parents of children with cancer of the central nervous system; 20 percent were parents of children with lymphomas; 12 percent were parents of children with bone cancer; 11 percent were parents with Wilms Tumor; 5 percent were parents of children with cancer of connective tissue; and 4 percent were parents of children with other cancers.

\section{Measures}

Extensive individual interviews lasting an hour and a half were conducted by trained psychology and sociology students to assess parents' experiences with, and 
reactions to, their child's illness. The interviews covered the following topics in both open-ended and structured formats.

1. History of the illness; informants' initial reactions and how they attempted to cope with the diagnosis and treatment.

2. How and what kind of information was shared with the child, siblings, extended family, friends, and school; effects of major events such as hospitalization, subsequent treatments, relapse, the child's return to school.

3. Relationship with the medical care organization; perceptions of the performance of physicians, doctors, and ancillary medical care staff; types of problems experienced in dealing with the medical care organization.

4. Effects of the illness on the family; changes in roles and task distributions in the family.

5. Sources of help and support in dealing with problems associated with the illness.

In addition to these interviews, a highly structured questionnaire with 3- or 5point rating scales was completed by parents and mailed back several days later. Ninety percent of the parents who were interviewed completed and returned the questionnaire. Data from the interviews were transcribed, and interview and questionnaire data were coded by several trained undergraduate and graduate raters.

Quality of Relationships with the Staff. Parents' responses to open-ended questions were used to derive ratings for seven dimensions of medical staff behavior. Trained coders used a 5 -point poor to excellent form $(5=$ excellent) to rate each dimension. The average intercoder agreement was 77 percent and the kappa estimate of intercoder reliability ranged from .52 to. 72 . Table 1 presents the correlation matrix for these seven dimensions of parent-medical staff relations. Most correlations are statistically significant but only moderately high. On this basis we believe they

TABLE 1

Correlation Matrix for Seven Dimensions of Relationships with the Medical Staff

$$
(\mathrm{N}=65)
$$

\section{1}

1. Information transmission

2. Communication (clarity, honesty)

3. Acceptance of parental efficacy

4. Conflict resolution

5. Empathy with child

6. Personal contact with staff

7. Staff competence

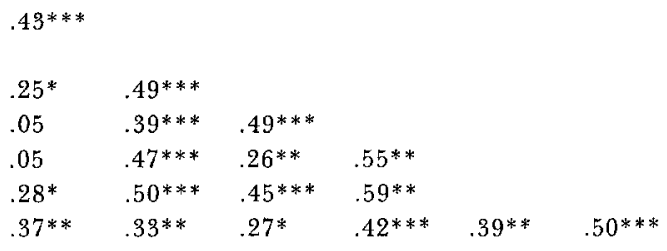

${ }^{*} \mathrm{p}=<.05$

${ }^{* *} \mathrm{p}=<.01$

${ }^{* * *} \mathrm{p}=<.001$

$\begin{array}{llllll}3 & 4 & 5 & 6 & 7\end{array}$


probably tap a common phenomenon, but also empirically refer to distinctive aspects of a global or general set of issues.

1. Information transmission refers to parents' assessment of the amount and regularity of information provided to them by the medical staff, especially regarding the outcome of diagnostic tests and the treatment plan (Inter-Coder Agreement $=90$ percent .

2. Communication (clarity and honesty) refers to parents' assessment of the openness and clarity with which the medical staff communicated with them about such complex or emotion-laden issues as the child's prognosis and the psychological consequences of treatment (Inter-Coder Agreement $=90$ percent).

3. Parental efficacy refers to parents' assessment of the extent to which the staff viewed them as knowledgeable about their child and dealt with them as collaborators in treating the child (Inter "Coder Agreement $=70$ percent).

4. Resolution of Conflicts refers to parents' assessment of the extent to which conflicts or disagreements were approached openly or directly by the staff and resolved in a harmonious and symmetrical manner (Inter-Coder Agreement $=70$ perecnt).

5. Empathy with the child refers to parents' assessment of the medical staff's personal concern and solicitude for the child's welfare and comfort (Inter-Coder Agreement $=70$ percent $)$.

6. Personal contact with staff refers to parents' assessment of the warmth, concern, and emotional closeness they personally experienced in their interactions with the staff (Inter-Coder Agreement $=70$ percent).

7. Staff competence refers to parents' assessment of the staff's technical knowledge and skill in providing high-quality care to their child. Parents' judgment of competence often revolved around their observations of the carefulness and completeness of diagnosis and treatment, the degree to which the different services were coordinated in providing care (radiation and hematology-oncology), the care with which IV's and medications were monitored, and the extent to which the medical staff was vigilant and or incisive in picking up and responding to symptoms or changes in the child's physical well-being (Inter-Coder Agreement $=80$ percent).

Satisfaction with the Medical Staff. These measures were based on questionnaire ratings made by parents of (a) changes in their views of the medical staff resulting from their experience with the illness, (b) stressors related to the medical system which parents experienced during the illness, and (c) sources of professional staff support they encountered or utilized.

1. Anger with medical staff-5-point ratings of changes in anger resulting from experience with the medical staff.

2. Respect for medical system-5-point rating of changes in respect resulting from experience with the medical staff.

3. Liking of medical staff-5-point rating of changes in positive feelings toward the medical staff resulting from the experience. 
4. Tenseness in relations with medical staff-3-point rating of stress resulting from tense relations with medical staff.

5. Helpfulness and support of medical staff-5-point rating of two items, one indicating helpfulness of doctors and the other of nurses.

6. Global satisfaction with the medical staff-a composite index of the anger, respect, liking, tenseness, and support ratings, which provides a global assessment of parents' experience and satisfaction with the medical staff (alpha estimate of internal consistency $=.77$ ).

\section{RESULTS}

\section{Staff Relationships and Parent Satisfaction}

In order to ascertain the relative importance of these seven dimensions of parents' relationship with the medical staff in predicting the quality of parents' experiences in medical care organizations, a series of multiple regressions were computed. The seven dimensions were used as predictors, and six different variables representing relationship outcomes were used as criteria.

Table 2 presents the statistical tests of significance for the regression equations computed for these relationships. Parental reports of tenseness in relating to the medical staff and increased liking of the staff were not predicted significantly by any of the relationship dimensions. The results of the four remaining regressions are significant and appear in Table 3. Parents' reports of staff relationships significantly predicted the composite index measuring parents' satisfaction with the medical staff, accounting for 37 percent of the variance of the total index. Of all the relationship dimensions, personal contact with the staff was the best single predictor of this composite measure of parental satisfaction (partial $r=+.32$ ).

The regression equation for increased anger toward medical staff also

TABLE 2

F-Value for Multiple Regression Equations

Predicting Aspects of Parental Satisfaction

\begin{tabular}{cc}
\hline Measures of Satisfaction & F-Value (df) \\
\hline Composite Satisfaction Index & $3.96^{*}(7,47)$ \\
Tenseness in Relationship & N.S. \\
Liking of Staff & N.S. \\
Increased Anger & $3.34^{*}(7,47)$ \\
Perception of Helpfulness & $3.44^{*}(7,66)$ \\
Increased Respect & $2.62^{*}(7,47)$
\end{tabular}

${ }^{*} \mathrm{P}=<.05$

N.S. = non-significant 
TABLE 3

Least Squares Regressions using parent-staff relationship dimensions as predictors of several measures of parent satisfaction with treatment (i.e., (a) composite index, (b) anger toward medical staff, (c) helpfulness of medical staff and (d) increased respect for medical profession).

A. Composite Index of Quality of Relationship with the Medical Care Staff

\begin{tabular}{lcccc}
\multicolumn{1}{c}{ VARIABLE } & $\begin{array}{c}\text { ZERO-ORDER } \\
\text { CORRELATION }\end{array}$ & $\begin{array}{c}\text { PARTIAL } \\
\text { CORRELATION }\end{array}$ & $\begin{array}{c}\text { BETA } \\
\text { COEFFICIENT }\end{array}$ & T-STATISTIC \\
\hline Information transmission & $(\mathrm{n}=65)$ & & & \\
Communication & +.22 & +.21 & +.95 & \\
Parental efficacy & $+.28^{*}$ & +.00 & -.01 \\
Conflict resolution & +.15 & -.16 & -1.05 & \\
Empathy with child & $+.36^{*}$ & +.18 & +1.19 \\
Personal contact & $+.36^{*}$ & +.12 & +.68 & \\
Staff competence & $+.44^{*}$ & +.32 & +1.8 & $2.3^{*}$
\end{tabular}

B. Increased Anger Toward Medical Staff

\begin{tabular}{lcccc} 
& ZERO-ORDER & PARTIAL & BETA & \\
VARIABLE & CORRELATION & CORRELATION & COEFFICIENT & T-STATISTIC \\
\hline
\end{tabular}

$\begin{array}{llll}\text { Information transmission } & +.03 & +.02 & +.02 \\ \text { Communication } & -.28^{*} & +.04 & -.04 \\ \text { Parental efficacy } & -.20 & -.16 & -.18 \\ \text { Conflict resolution } & -.40^{*} & -.21 & -.25 \\ \text { Empathy with child } & -.45^{*} & -.24 & -.26 \\ \text { Personal contact } & -.42^{*} & -.23 & -.24 \\ \text { Staff competence } & -.08^{*} & +.19 & +.15\end{array}$

C. Helpfulness of Medical Staff

\begin{tabular}{ccccc} 
& ZERO-ORDER & PARTIAL & BETA & \\
VARIABLE & CORRELATION & CORRELATION & COEFFICIENT & T-STATISTIC \\
\hline
\end{tabular}

$\begin{array}{llccc}\text { Information transmission } & +.25^{*} & +.22 & +.56 & 1.9^{*} \\ \text { Communication } & +.06 & -.10 & -.30 & \\ \text { Parental efficacy } & -.15 & +.44 & -1.4 & 4.0^{*} \\ \text { Conflict resolution } & -.02 & +.17 & +.57 & \\ \text { Empathy with child } & +.08 & +.04 & +.12 & \\ \text { Personal contact } & +.12 & +.04 & +.12 & \\ \text { Staff competence } & +.28^{*} & +.18 & +.47 & \end{array}$

D. Increased Respect for the Medical System

\begin{tabular}{|c|c|c|c|c|}
\hline VARIABLE & $\begin{array}{c}\text { ZERO-ORDER } \\
\text { CORRELATION }\end{array}$ & $\begin{array}{c}\text { PARTIAL } \\
\text { CORRELATION }\end{array}$ & $\begin{array}{c}\text { BETA } \\
\text { COEFFICIENT }\end{array}$ & T-STATISTIC \\
\hline & $(n=65)$ & & & \\
\hline Information transmission & +.23 & +.34 & +.32 & $2.7^{*}$ \\
\hline Communication & +.15 & +.12 & -.12 & \\
\hline Parental efficacy & +.04 & -.16 & -.19 & \\
\hline Conflict resolution & -.25 & +.24 & +.29 & \\
\hline Empathy with child & $+.30^{*}$ & +.18 & +.19 & \\
\hline Personal contact & $+.29 *$ & +.19 & +.19 & \\
\hline Staff competence & +.05 & +.26 & +.21 & $1.8^{*}$ \\
\hline
\end{tabular}

${ }^{*} \mathrm{p}=<.05$ 
is statistically significant, and accounts for 33 percent of the variance. Among the seven dimensions, a perceived lack of staff empathy with the child was the most critical determinant of anger (partial $r=-.24)$. Increased parental anger also was predicted by a lack of personal contact (zero-order $r=-.42$ ) and inadequate conflict resolution (zero-order $r=-.40$ ) behavior by the staff. However, when the variance these latter dimensions have in common with lack of empathy was partialed out, their relationships were nonsignificant.

Parents' assessments of the helpfulness of the medical staff (doctors and nurses) also was predicted significantly by these relationship dimensions, and 27 percent of the variance is thus accounted for. Perceptions of the helpfulness of the staff was related most highly to the staff's acceptance of parental efficacy (partial $r=+.22$ ).

Finally, increased respect for the medical system was predicted significantly by the relationship dimensions. Those aspects of staff behavior most highly related with increased parental respect for the medical system were information transmission (partial $r=+.34$ ) and staff competence (partial $r=+.26)$.

\section{DISGUSSION}

This research has attempted to advance our understanding of the multiple factors affecting the relationship between medical providers and parents of chronically and seriously ill children. We have gone beyond global statements of satisfaction with the staff and with staff-parent relationships to assess specific links among these factors. The diverse patterns emerging from the data support our contention that various dimensions of parent-staff relationships link in complex ways to parents' reactions to their experiences in medical care organizations. Specifically, the quality of parents' personal contact with doctors, that is, the sympathy, warmth, concern, and attention shown to parents as individuals, was most highly related to general satisfaction with the medical staff. These findings do not stand alone: Gordon ${ }^{12}$ also has pointed out how important such contact is for parents of sick children, noting that "parents need as much sympathetic understanding as their child" (p. 28).

The degree of increased anger parents reported was best related to their perceptions of the staff's empathy with the child. Parents were more likely to have greater anger with the medical staff when they questioned the staff's concern for or sensitivity to their child's special needs. Featherstone, ${ }^{13}$ writing about her own reactions to the medical staff's treatment of her handicapped child, also has emphasized how important it is for parents to feel that doctors respect and like their child. Since one's child is a reflection of oneself, and since an ill child is especially vulnerable, this dimension may have double-barelled impact on parents.

Increased respect for the medical care staff was most strongly related to 
parents' perceptions of the staff's transmission of information and to their evaluation of the staff's technical competence. This finding suggests that the respect and esteem parents accorded physicians was a result of the staff's perceived knowledge and skills with respect to diagnosis and treatment of the child and their ability and willingness to convey this information in a regular and meaningful fashion. Although parents ultimately may base their judgments of staff competence on the medical survival of their child, most parents of children with cancer understand that even the best available treatments may not help. In these instances parents' judgments regarding staff skills are based of social cues gleaned from their formal conferences and informal conversations. It is at these times that physicians make good or poor impressions or communicate that they do or do not know what they are doing. ${ }^{14}$

Parents' feelings of the staff's helpfulness was most highly related to their perception of the staff's transmission of information and acceptance of parental efficacy. When physicians, nurses, and other medical staff members failed to provide adequate information about treatment and prognosis, and when they did not involve parents in decision-making and care-taking tasks, parents were less likely to feel helped and supported, ${ }^{5,15-17}$. In other recent research on the medical staff relationships of parents of children with cancer, Mulhern, Crisco, and Camitta ${ }^{9}$ also have noted the importance of information transmission. Despite attempts to standardize the information doctors presented, these researchers found misunderstanding and disagreement common between mothers and fathers of ill children and their doctors. Moreover, the exclusion of parents from participative or efficacious roles in the care of their seriously ill children has been reported to contribute to parents' feelings of loss of control and helplessness. ${ }^{5,11,18}$

\section{CONCLUSION}

Childhood cancer is recognized widely as a crisis, both for parents and for their children. The medical staff plays a central role in this crisis and more generally in the lives of parents of children with cancer. They not only treat the children, hopefully with medical success, but they also provide (or fail to provide) important psychosocial supports that may help mediate the effects of this crisis on patients and parents. The complex and concrete dimensions of satisfactory relations discussed here indicate specific medical behaviors that are related to important outcomes for parents, such as feelings of anger, helpfulness, and respect. Hopefully, they take us beyond the global findings and platitudes that fail to provide understanding and therefore fail to guide or redirect specific staff behaviors. Rather than discussing broadly good relationships with the medical staff, we can now pinpoint the importance of in- 
formation transmission compared with full and honest communication and can distinguish between the staff's personal contact with parents and their empathic relationship with the child. Moreover, rather than discussing general parent satisfaction with medical care, we can make distinctions among parents' feelings of staff helpfulness and respect for the staff, between tenseness and anger, and so on.

Of the seven different dimensions of staff relationships identified by parents, information transmission and personal contact can be singled out for special emphasis. Personal contact stands out because it has the highest mean intercorrelation with parents' perceptions of other positive staff behaviors. Information transmission stands out because it predicts independently more of the individual satisfaction measures than any other single behavior. Although most medical professionals acknowledge the importance of these issues, they are easily overlooked and assigned lower priority when the staff is confronted with a family in crisis and with life and death problems. Perhaps with greater knowledge of the specific behaviors and perceptions involved, the medical staff can act to increase parents' positive evaluations and satisfaction with care. Perhaps with greater wisdom about the correlates of satisfaction with medical care, parents, too, can help construct more positive relationships. The achievement of this mutual agenda is obviously important for patients' and parents' quality of life during the course of children's treatment, and thereafter.

\section{REFERENCES}

1. Becker MH, Maiman LA: Strategies for enhancing compliance. Community Health 6:113-135, 1980.

2. McArthur R, Tomm K, Leahey M: Management of diabetes mellitus in children. Can Med Ass $J$ 114:783-787, 1976.

3. DiMatteo MR, Hayes R: The significance of patients' perceptions of physician conduct: A study of patient satisfaction in a family practice center. Community Health 6:18-34, 1980.

4. DiMatteo MR, Prince L, Taranta A: Patients' perceptions of perceptions of physicians' behaviors: Determinants of patients' commitment to the theapeutic relationship.J Community Health 4:280-290, 1979 .

5. Adams D: Childhood Malignancy: The Psychosocial Care of the Child and Family. Springfield, Ill.; Thomas, 1979 .

6. Davis MS: Variations in patients' compliance with doctors' advice: An empirical analysis of patterns of communication. AmerJ Pub Health, 58:274-288, 1968.

7. Schulman BA: Active patient orientation and outcomes in hypertensive treatment: Application of a socio-organizational perspective. Med Care, 17:267-280, 1979.

8. Francis V, Korsch BM, Morris MJ: Gaps in doctor-patient communication Patients' reponse to medical advice. $N$ Eng J Med, 280:535-540, 1969.

9. Rath A: Parents perceptions of nursing care of their chronically ill child. In Azarnoff P, Hardgrove C (eds): The Family in Child Health Care. New York, Wiley, 1981.

10. Chesler MA, Barbarin OA: Issues between the medical staff and parents of children with cancer: Parents' perspective of institutionalized relationships (submitted for publication).

11. Chesler MA, Barbarin OA, Chesler JE, Hughes D, Lebo J: Role of informal networks and medical care organizations in helping families cope with childhood cancer: Prospects for collaboration (CRSO Working Paper \#243). University of Michigan, Center for Research on Social Organization, 1981.

12. Gordon, B: The familiar stranger. In Azarnoff P, Hardgrove C (eds): The Family in Child Health Care. New York, Wiley, 1981. 
13. Featherstone $\mathrm{H}$ : A Difference in the Family. New York, Basic Books, 1980.

14. Mechanic D; The influence of mothers on their children's health attitudes and behaviors. Pediatrics, 33:444-453, 1964.

15. Futterman E, and Hoffman I: Crisis and adaptation in the families of fatally ill children. In Anthony J, Koupernick C (ed.): The Child in The Family: The Impact of Death and Disease, Vol.1, New York, Wiley, 1973.

16. McCollum A, Schwartz A: Social work and the mourning patient. Social Work 17:25-36, 1972.

17. Mulhern R, Crisco J, Camitta B: Patterns of communication among pediatric patients with leukemia, parents and physicians: Prognostic disagreements and misunderstandings. $J$ Ped 99:(3), 480-483, 1981.

18. Richmond J, Waisman H: Psychologic aspects of management of children with malignant diseases. Amer J Diseases of Children 89:42-47, 1955.

19. Hamburg D, Adams J: A perspective on coping behavior: Seeking and utilizing information in major transitions. Arch General Psychiatry 17:279, 1967.

20. Kulka B, Cupper L, Cassel J, Mayo F: Doctor-patient communication and outcomes among diabetic patients. J Community Health 1:15-27, 1975. 